\title{
ANÁLISE DE SINAIS ELETROMIOGRÁFICOS DO MÚSCULO TEMPORAL UTILIZANDO TRANSFORMADA WAVELETS
}

\section{ANALYSIS OF THE TEMPORAL MUSCLE ELECTROMYOGRAPHIC SIGNALS USING WAVELETS}

\author{
Ingrid Solange Sepúlveda Muñoz ${ }^{1}$ \\ Alberto José Carvalho Martins Motta ${ }^{2}$ \\ Renata Amadei Nicolau ${ }^{3}$ \\ Carlos Alberto Kelencz ${ }^{4}$ \\ Alderico Rodrigues de Paula Júnior ${ }^{5}$
}

RESUMO: A Transformada Wavelet (TW) tem sido aplicada em diversas áreas biológicas. Visando verificar a eficiência da TW em sinais biológicos, o presente estudo apresenta uma técnica de analisar sinais eletromiográficos (EMG) do músculo temporal em contração isométrica máxima e durante o processo de fadiga, utilizando a TW. Participaram do experimento 20 voluntários do gênero feminino. Estes foram submetidos a uma contração isométrica máxima dos músculos temporal direito e esquerdo durante 60 segundos, utilizando uma plataforma oclusal. Para analisar aplicou-se a TW nos dados obtidos, com o auxílio do programa desenvolvido para o MatLab 7.0.1., usando como base a TW de Morlet. Nos resultados obtidos, verificou-se que, no sinal do músculo temporal direito pré-fadiga, houve um valor significativo total da potência (VSTP) em 50\% da população avaliada. Com relação à musculatura esquerda, esta apresentou VSTP em 45\% dos voluntários. No músculo temporal direito e esquerdo, após o início da fadiga, observou-se presença de VSTP em $85 \%$ e $90 \%$ das voluntárias, respectivamente. Esta pesquisa permitiu concluir que a TW pode ser uma importante ferramenta no processamento e análise de sinais biológicos, permitindo uma avaliação de tempo e frequência do desempenho do músculo estudado. Foi possível compactar os dados brutos dos sinais reais, obtendo, dessa forma, detalhes importantes do músculo estudado.

Palavras-chave: Transformada Wavelet; eletromiografia; fadiga muscular.

ABSTRACT: The Wavelet Transform (WT) has been applied in diverse areas of biology. In order to verify the efficiency of WT in biological signs, this study presents a technique to analyze electromyography signals during maximum isometric contraction of the temporal muscle and during muscle fatigue, processed by WT. Twenty female volunteers were submitted to the maximum isometric contraction of the right and left temporal muscles for 60 seconds using a mandibular force transducer. WT was applied to the data through the software MatLab 6.1 based on the Morlet WT. The results showed that in the pre-fatigue signal, the right temporal muscle had a value of total significant power (VSTP) in 50\% in the evaluated population. However, the left pre-fatigue muscle had a VSTP in $45 \%$ of the volunteers. In the left and right temporal muscle after fatigue, the presence of VSTP was observed in $85 \%$ and $90 \%$ of the volunteers, respectively. This research leads to the conclusion that the WT may be an important tool in processing and analyzing biological signals, providing a time-frequency analysis of the studied muscle performance. It was possible to compact the raw data from real signs, thereby obtaining important details of the studied muscle.

Keywords: Wavelet Transform; electromyography; muscular fatigue.

\footnotetext{
1 Doutoranda em Engenharia Biomédica - Universidade do Vale do Paraíba - Univap. E-mail: professoraingrid2009@hotmail.com.

${ }^{2}$ Mestre em Engenharia Biomédica - Univap. E-mail: bebetomotta@yahoo.com.br.

${ }^{3}$ Doutora em Engenharia Biomédica - Univap e Professora Titular da Univap. E-mail: rani@univap.br.

4 Doutor em Engenharia Biomédica - Univap e Professor Titular do Centro Universitário Ítalo Brasileiro. E-mail: carloskelencz@terra.com.br.

${ }^{5} \mathrm{PhD}$. in Computer Science - UCLA e Professor Titular da Univap. E-mail: alderico@univap.br.
} 


\section{INTRODUÇÃO}

Nos últimos anos, a eletromiografia de superfície tornou-se uma técnica utilizada em diferentes áreas do conhecimento humano. Por ser uma técnica de fácil utilização e não invasiva, pode ser empregada no monitoramento da atividade elétrica muscular (GONZÁLEZ et al., 2009).

A Raiz Quadrática Média (RMS) pode ser utilizada como ferramenta matemática para análise de sinais eletromiográficos (EMG), no domínio do tempo (BASMAJIAN; DE LUCA, 1985; GONZÁLEZ et al., 2009).

A partir da análise dos valores RMS, podem ser obtidas informações sobre a ativação muscular, intensidade de contração e processo de fadiga (GONÇALVES; SILVA, 2007). Essa técnica permite a visualização gráfica da atividade elétrica muscular em função do tempo, mas não provê informações espectrais. Já, a transformada de Fourier fornece informações espectrais. Contudo, nenhuma das técnicas de análises permite avaliação do sinal correlacionando tempo e frequência, além de comumente apresentar elevado nível de ruído.

A TW pode ser empregada para a análise de sinais biológicos, pois permite a correlação de tempo e frequência (GONZÁLEZ et al., 2009; KUMAR; PAH; BRADLEY, 2003; MUÑOZ, 2009; PINTO NETO; MAGINI, 2008). Tal técnica pode ser aplicada à análise de sinais EMG (GONZÁLEZ et al., 2009; KUMAR; PAH; BRADLEY, 2003).

Dentre os diferentes tipos de TW, a de Morlet mostra-se adequada para a análise de sinais EMG (PINTO NETO; MAGINI, 2008; MUÑOZ, 2009). O termo Wavelets refere-se a um conjunto de funções com forma de pequenas ondas geradas por dilatações, $\psi(\mathrm{t}) \rightarrow \psi($ at), e translações, $\psi(\mathrm{t}) \rightarrow \psi(\mathrm{t}+\mathrm{d})$ sendo a e $\mathrm{d}$ números reais, de uma função base $\psi(\mathrm{t})$, a Wavelet-mãe (BOLZAN, 2004; BARBOSA; BLITZKOW, 2008). Após a geração dos coeficientes Wavelets, o cálculo do Espectro Wavelet Global (GWS - Global Wavelet Spectrum) pode ser obtido. O GWS é a representação gráfica da média espectral das frequências em janelas selecionadas de tempo (PINTO NETO; MAGINI, 2008; MOTTA, 2009; MUÑOZ, 2009).

Na geração do GWS, um escalograma é obtido, permitindo a identificação de ganho ou perda de rendimento muscular por meio de oscilações da intensidade de frequência do sinal (BOLZAN, 2004; MOTTA, 2009; MUÑOZ, 2009). A partir do GWS também pode ser extraído o Valor Significativo Total da Potência (VSTP), dado estatístico das oscilações de frequência mais representativas (TORRENCE; COMPO, 1998).

Considerando as possibilidades de utilização de novas ferramentas matemáticas na análise de sinais biológicos, o presente estudo objetivou testar a aplicabilidade da TW na avaliação de sinais EMG do músculo temporal.

\section{METODOLOGIA}

$\mathrm{Na}$ primeira etapa do trabalho, foram analisados 20 voluntários, encaminhados da Policlínica Odontológica da Universidade do Vale do Paraíba (UNIVAP), do gênero feminino, destros, $24 \pm 6$ anos, com todos os elementos dentais e não possuindo desordens temporomandibulares. Este estudo foi aprovado pelo Comitê de Ética do Instituto de Pesquisa \& Desenvolvimento da UNIVAP, sob o protocolo H076/2006/CEP.

Para a indução da fadiga muscular 
(FM), foi utilizada uma plataforma oclusal (EMG System do Brasil Ltda - Fig. 1), de borracha de $12 \mathrm{~mm}$ de largura e $4 \mathrm{~mm}$ de espessura, entre os dentes molares, limitando o fechamento da boca bilateralmente (dinamômetro).

Foram utilizados dois eletrodos de superfície bipolares ativos com contato de prata, com pré-amplificação do sinal em 20 vezes para a captação dos sinais EMG. Os eletrodos foram posicionados nos músculos temporais, entre o ponto motor e a junção miotendinosa do feixe anterior (DELAGI, 2005), permanecendo paralelos em direção às fibras musculares com as barras de prata perpendiculares às fibras (VITTI; BASMAJIAN, 1977). Previamente à colocação dos eletrodos no músculo temporal, realizou-se a tricotomia e fricção da pele com algodão embebido em álcool 70\%
(CALIFANO, 2006; SALOMONOW, 1995).

Um eletrodo de referência foi posicionado no processo estilóide da ulna direita. Todos os procedimentos relativos à coleta, ao registro e ao tratamento do sinal EMG, foram seguidos de acordo com recomendações da Sociedade Internacional de Eletrofisilogia e Cinesiologia (ISEK).

Para o registro dos sinais EMG, cada voluntário foi posicionado sentado, em frente ao transdutor de mordida, para o ajuste da altura entre a plataforma oclusal e a arcada dentária (Fig. 1), observando que os dentes estivessem localizados acima de $2,0 \mathrm{~mm}$, na barra de mensuração. Por meio de comando verbal, cada voluntário foi orientado a realizar atividade isométrica de mordida sobre a plataforma oclusal, por 60 segundos (MUÑOZ et al., 2009; CALIFANO et al., 2009; KELENCZ et al., 2010).

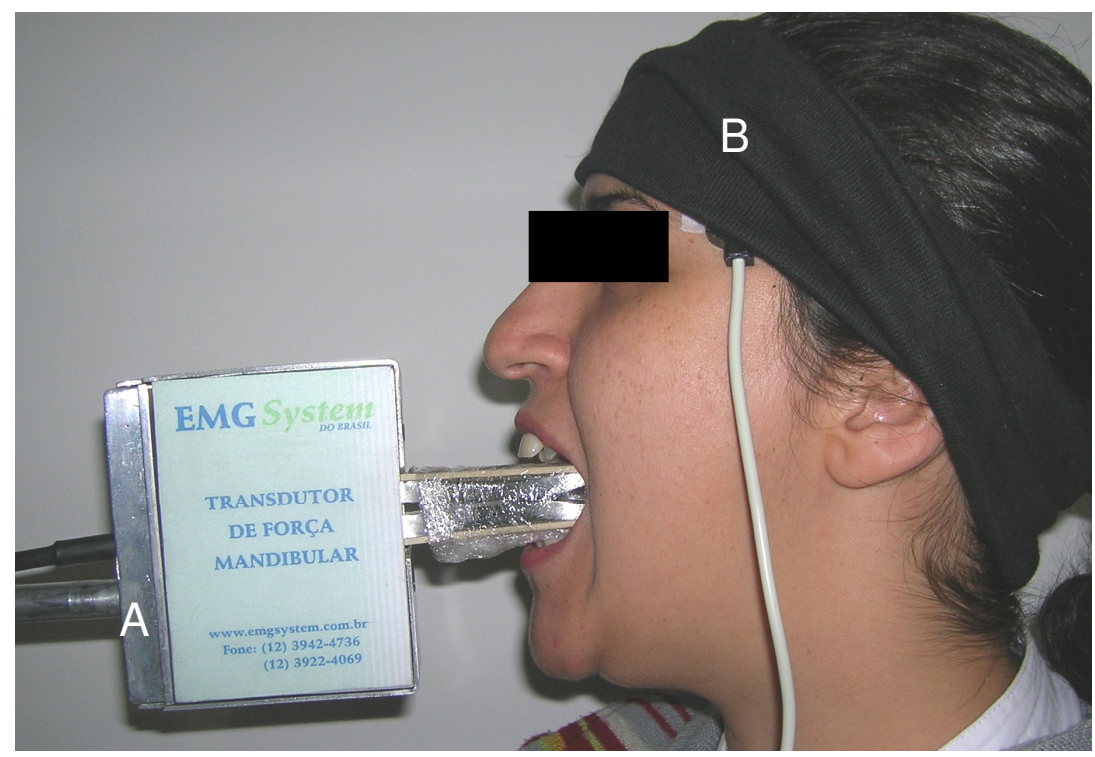

Fig. 1 - Voluntária durante a aquisição dos sinais de eletromiografia. A - Plataforma oclusal sobre a posição, durante o processo de geração de fadiga. B - Eletrodos.

Para a captação dos sinais EMG, utilizou-se o sistema de eletromiografia, de 16 canais, da marca EMG System do Brasil
Ltda, com ganho de amplificação de 1000 vezes, filtro analógico passa banda de 20 a $500 \mathrm{~Hz}$ e rejeição de modo comum > 120 
dB. Os sinais EMG foram captados com uma frequência de $1000 \mathrm{~Hz}$ (1000 amostras por segundo), digitalizados por uma placa de conversão $A / D$ de 16 bits, faixa de entrada de \pm 5 Volts e com precisão de $\pm 5000 \mu \mathrm{V}$.

A partir dos sinais EMG e com o auxílio do programa Matlab 7.0.1., foram obtidos escalogramas. Para tal, empregouse a função "extrairr", utilizada para extrair o sinal digitalizado, gerando um vetor de dados (BOLZAN, 2004; DELFINO, 2006; MUÑOZ, 2009).

Para a obtenção do tempo de início do processo de fadiga, foi plotado o gráfico de força, por meio do programa EMGWorks
Analysis, e identificado o maior pico de força, e calculou-se o decaimento de $20 \%$ dessa força, chegando, dessa forma, ao tempo inicial do processo de fadiga muscular (TRIBESS; VIRTUOSO JR., 2005; KELENCZ et al., 2010).

Baseado no tempo total de coleta ( 60 segundos), a fadiga muscular foi analisada considerando o sinal inteiro, o sinal pré fadiga e o sinal pós fadiga (Fig. 2). Do tempo total de coleta foram gerados escalogramas, contendo informações, como a frequência $(\mathrm{Hz})$, tempo (s) e energia $\left(\mu \mathrm{V}^{2}\right)$. Dos escalogramas foi possível obter o GWS, e, a partir deste, o VSTP pré e pós fadiga muscular.

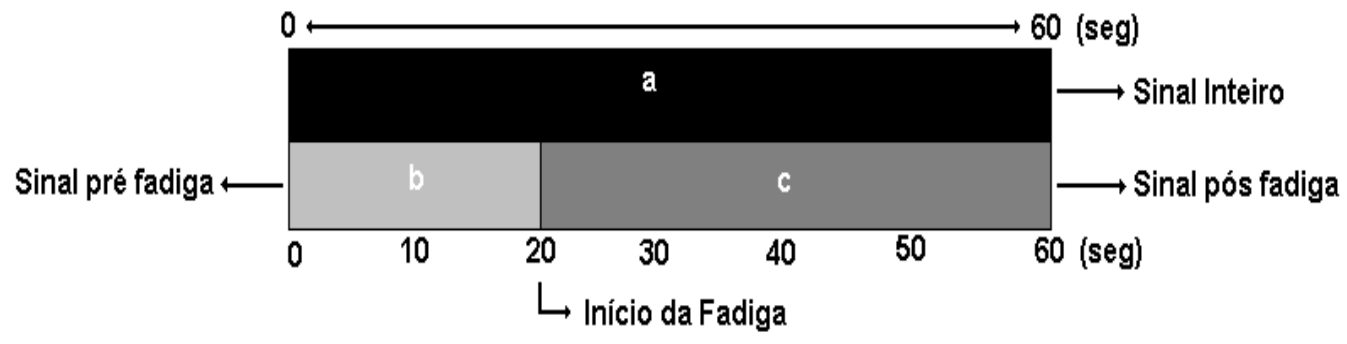

Fig. 2 - Exemplo de corte de um sinal: a) Sinal inteiro de 0 a 60 segundos; b) Sinal pré fadiga de 0 a 20 segundos; c) Sinal pós fadiga de 20 a 60 segundos.

\section{RESULTADOS}

A análise dos sinais, obtidos a partir temporal, para a determinação de perfil de dos escalogramas, permitiu identificar comportamento/atividade muscular dos vantagens, desvantagens, abrangência e voluntários estudados (Fig. 3). limitações da aplicação da TW em músculo 


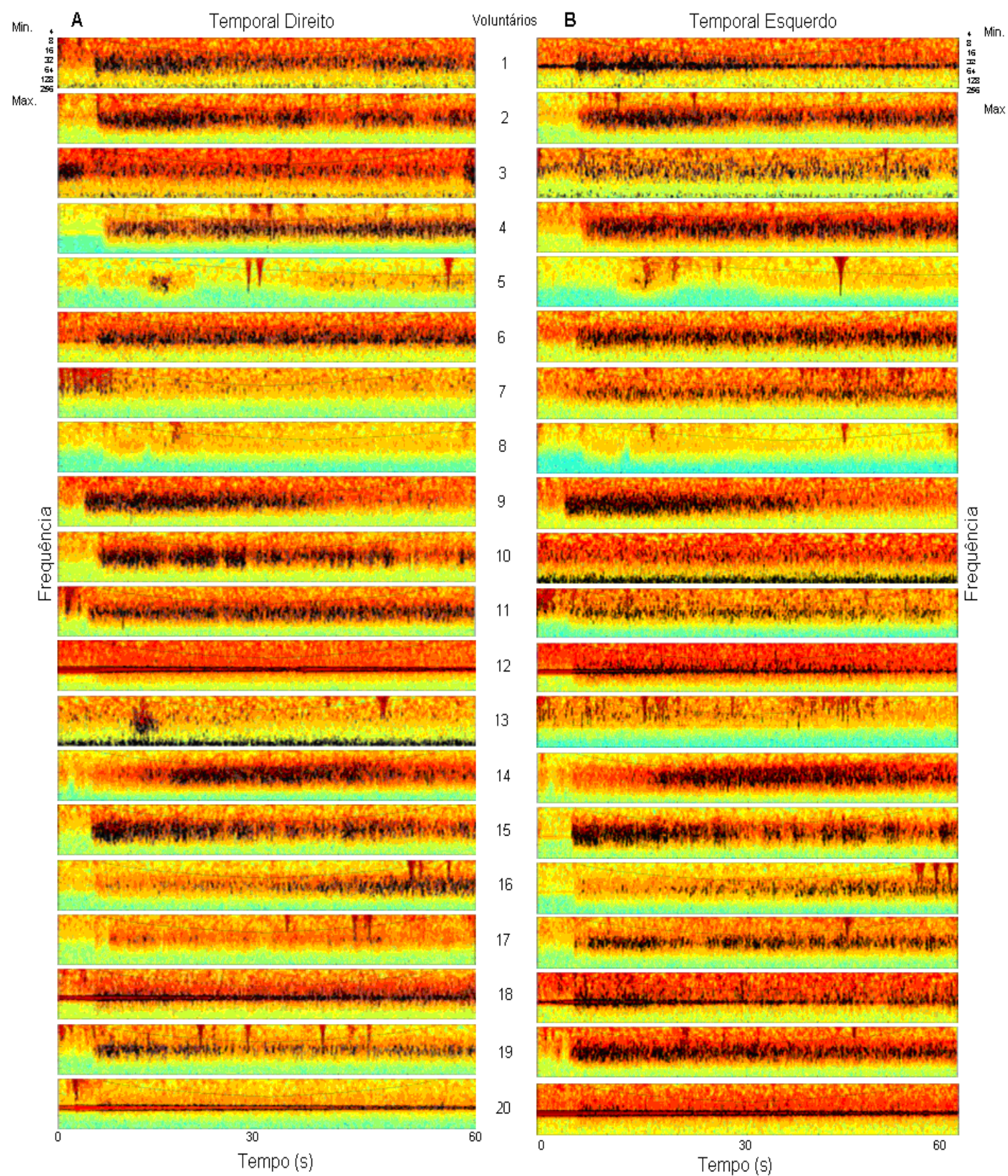

Fig. 3 - Escalogramas extraídos da Transformada Wavelet, do sinal EMG do músculo temporais direito (A) e esquerdo (B) dos 20 voluntários.

Utilizando a análise qualitativa dos escalogramas (Fig. 3), foi possível observar zonas de alta intensidade de energia (ZAIE) nos voluntários estudados, com exceção dos voluntários 5 e 8, os quais apresentaram ausência de ZAIE. Observou-se um comportamento semelhante, entre o sinal do músculo temporal direito e temporal esquerdo, na maioria dos voluntários.

O início da fadiga pode ser percebido nas áreas negras, nos escalogramas, as quais apresentaram redução da intensidade de energia abaixo de $20 \%$ dos valores iniciais. Exemplo desse comportamento pode ser evidenciado nos escalogramas dos voluntários 9, 10, 14, 15 (Fig. 3). 
Nos escalogramas, no tempo inicial das coletas (entre 0 e 10 segundos), dos voluntários 1, 2, 4, 6-10, 14-17, 19 (Fig. $3)$, pode-se notar que não houve nenhum sinal elétrico referente à contração muscular. O voluntário 16 apresentou baixa intensidade de sinal até 30 segundos de atividade isométrica. Após esse tempo. as ZAIE aumentaram (Fig. 3).

Dos dados obtidos do sinal EMG dos músculos temporais, por intermédio da TW, foram extraídos os VSTPs em $\mu \mathrm{V}^{2}$ (Tabela 1). Considerando que o VSTP é obtido a partir do GWS, e que se caracteriza pelo resultado estatístico da existência ou não de oscilações significativas de frequências durante a atividade muscular (TORRENCE; COMPO, 1998), pode-se verificar que não existe correlação entre o tempo de fadiga e a presença ou não dessa significância (Tabela 1). A mesma constatação pode ser atribuída ao músculo direito e esquerdo pré fadiga, nos quais o VSTP não pode ser observado em todos os indivíduos.

Pôde-se verificar, no sinal do músculo temporal direito pré fadiga, que o VSTP estava presente em $50 \%$ dos indivíduos. $\mathrm{Na}$ análise da musculatura esquerda pré fadiga observou-se o VSTP em 45\% dos voluntários. Contudo, observa-se a presença expressiva de VSTP pós fadiga em ambos os lados estudados (musculatura direita e esquerda), ou seja, mais oscilações de frequência no GWS. No músculo temporal direito e esquerdo, após início da fadiga, a presença de VSTP ocorreu em 85\% e 90\% dos voluntários, respectivamente.

Tabela 1- Valor Significativo Total do Músculo Temporal (pré/pós fadiga)

\begin{tabular}{|c|c|c|c|c|c|}
\hline \multirow[t]{2}{*}{ Voluntários } & \multirow[t]{2}{*}{ TIF } & \multicolumn{2}{|c|}{$\begin{array}{c}\text { Músculo temporal direito } \\
\operatorname{VSTP}\left(\mu V^{2}\right)\end{array}$} & \multicolumn{2}{|c|}{$\begin{array}{c}\text { Músculo temporal esquerdo } \\
\text { VSTP }\left(\mu V^{2}\right)\end{array}$} \\
\hline & & TDPF & TDPoF & TEPF & TEPoF \\
\hline 7 & 30 & 133290 & 4597 & 4036 & 4938 \\
\hline 2 & 20 & - & 21263 & - & 10155 \\
\hline 3 & 40 & 45779 & 9729 & 391130 & 15381 \\
\hline 4 & 33 & - & 6263 & - & 4106 \\
\hline 5 & 10 & - & - & - & - \\
\hline 6 & 20 & 802 & 24106 & - & 3290 \\
\hline 7 & 35 & 335220 & - & - & 2385 \\
\hline 8 & 11 & - & - & 9844 & - \\
\hline 9 & 40 & - & 37611 & - & 19242 \\
\hline 10 & 39 & - & 1376 & 2690 & 4923 \\
\hline 11 & 24 & 1849 & 12646 & 286150 & 4783 \\
\hline 12 & 40 & 778 & 6639 & 7780 & 18446 \\
\hline 13 & 10 & 3370 & 6200 & 114580 & 841 \\
\hline 14 & 41 & - & 55488 & - & 56468 \\
\hline 15 & 31 & - & 59209 & - & 56257 \\
\hline 16 & 13 & - & 2310 & - & 900 \\
\hline 17 & 24 & - & 249 & - & 3507 \\
\hline 18 & 25 & 17694 & 9828 & 8623 & 8093 \\
\hline 19 & 25 & 160020 & 16326 & - & 9197 \\
\hline 20 & 14 & 30211 & 3663 & 42617 & 14061 \\
\hline
\end{tabular}

TIF - Tempo de início da fadiga; TDPF - Temporal direito pré fadiga; TDPoF - Temporal direito pós fadiga; TEPF - Temporal esquerdo pré fadiga; TEPoF - Temporal esquerdo pós fadiga, VSTP SigTotal - Valor significativo total da potência $\left(\mu V^{2}\right)$. 


\section{DISCUSSÃO}

Dentre as formas de captação e análise do sinal elétrico do músculo temporal, a mais conhecida e utilizada é a eletromiografia (CALIFANO et al., 2009; SHINOZAKI, 2006). A análise de sinais EMG permite a visualização do sinal elétrico capturado em tempo real. Contudo, os sinais biológicos geram, comumente, arquivos da ordem de gigabits, levando a dificuldades no armazenamento e processamento, com elevado gasto de tempo. Existe, ainda, a inviabilidade de avaliação quantitativa panorâmica do sinal, devido à sua extensão.

A compressão dos dados EMG não permitem visualização de zonais de maior intensidade de atividade muscular (KUMAR; PAH; BRADLEY, 2003; MOTTA, 2009; MUÑOZ, 2009).

Estudos realizados com eletromiografia, em musculatura temporal (SHINOZAKI, 2006; CALIFANO et al., 2009; MOTTA, 2009), demonstram a limitação dessa forma de análise, pois o detalhamento da potência de sinal, em relação ao tempo de atividade muscular e ganho, não pode ser observado. Dessa forma, no presente estudo, a atividade do músculo temporal foi avaliada com o emprego de TW, a partir de sinais EMG.

A TW permitiu a observação de níveis de intensidade de atividade muscular direita e esquerda, pré e pós-fadiga em voluntários sadios por meio de escalogramas (Fig. 3, Tabela 1).

Na maioria dos escalogramas (Fig. 3), a ZAIE pode ser observada do início da fadiga até o final da coleta. Muitos dos escalogramas não apresentam sinal evidente nos momentos iniciais de atividade (pré-fadiga), acentuando-se gradualmente até o início da fadiga. Essa inconsistência gráfica do sinal (ex. voluntário 16, Fig. 3) pode ser devida à falha do comando verbal (interpretação falha pelos voluntários), alterações no recrutamento de acordo com exercício isométrico (60 segundos) para o início da coleta.

A presença de baixa ou nenhuma intensidade de sinal, nos primeiros cinco segundos da coleta, pode ter ocorrido devido à falha de sincronização entre o início da aquisição dos sinais e o início no comando verbal ou demora na compressão da plataforma oclusal (MOTTA, 2009). Podese sugerir uma mudança na metodologia de aquisição de sinais, em nível clínico e/ou experimental, iniciando-se a contagem do tempo apenas no momento em que iniciar a captação do sinal ZAIE e não antes disso. A regularidade dos sinais EMG, nos escalogramas, poderá ser otimizada com a implementação de um sistema de Biofeedback.

As variações no início de ZAIE, durante atividade muscular, não foram observadas em estudos anteriores empregando músculo masseter, demonstrando características específicas existentes em diferentes músculos da face (MUÑOZ, 2009). As características fisiológicas dos diferentes músculos da face podem justificar tal comportamento variado nos sinais (BIASSOTO; BIASOTTOGONZALEZ; PANHOCA, 2005; DRAKE et al., 2005; NOZAKI et al., 2010).

O decaimento da força, que ocorreu simultaneamente ao início da fadiga, nos voluntários, pode ser percebido nos escalogramas (Fig. 3) por redução das ZAIEs. Nesse momento, ocorre uma diminuição no recrutamento das fibras musculares, e, consequentemente, uma 
diminuição na intensidade do sinal (ABDULLAH et al., 2010). Esse fato pode ser observado em $55 \%$ dos voluntários. Em aplicações clínicas, esse tipo de diagnóstico poderia indicar ganho ou perda de rendimento muscular.

Foi possível observar grande percentual de voluntários com ZAIEs e VSTP após o início da fadiga muscular de ambos os lados (direito e esquerdo, Fig. 3 e Tabela 1). Esses dados indicam maior recrutamento de fibras musculares para compensar a diminuição da força de contração e tentativa de manutenção do nível de tensão das fibras fadigadas (MORITANI et al., 1993). Esse comportamento também pode ser observado pelo aumento do sinal EMG (MIYASHITA et al., 1981; HANNINEN et al., 1989), contudo, sem o detalhamento obtido com a TW (intensidade em ZAIEs e VSTP).

Considerando que o VSTP refere-se às oscilações de frequência durante atividade muscular, é possível sugerir que essa oscilação ocorre, de forma significativa, após exercício isométrico do músculo temporal. A TW poderia auxiliar no diagnóstico e acompanhamento pós-terapia odontológica e/ou fisioterapêutica de tais indivíduos com desordens temporomandibulares (DTM). Indivíduos com DTM comumente apresentam hiperatividade dos músculos mastigatórios.

\section{CONCLUSÃO}

Concluiu-se que a TW é uma ferramenta importante que pode ser empregada no processamento de sinais EMG do músculo Temporal em contração isométrica. A aplicação da TW permitiu minimizar dados brutos dos sinais por meio de compressão, viabilizando a análise detalhada da atividade do músculo Temporal. A intensidade de força vs tempo de atividade muscular, momento da atividade e/ou fadiga, presença ou não de lado predominante, caracterizaram os dados mais importantes obtidos por meio da ferramenta estudada.

\section{AGRADECIMENTOS}

I. S. S. Muñoz agrade à CAPES/PROSUP, pela bolsa de doutorado. R. A. Nicolau agradece ao CNPq, pela bolsa de Produtividade (Processo 314455/2009-4). Os autores agradecem à EMG System do Brasil Ltda, pela concessão de equipamentos, e à bibliotecária Rúbia Gravito Gomes (UNIVAP).

\section{REFERÊNCIAS}

ABDULLAH, S. et al. The Morlet wavelet analysis for fatigue feature clustering. WSEAS Transactions on Mathematics, v. 9, n. 5, p. 345-354, May 2010.

BARBOSA, A. C. B.; BLITZKOW, D. Ondaletas: Histórico e Aplicação. São Paulo: IAG/USP, 2008.

BASMAJIAN, J. V.; DE LUCA, C. J. Muscle alive: their function revealed by eletromyography. 5. ed. Baltimore: Williams Wilkins, 1985. p. 501-561.

BIASOTTO, D. C.; BIASOTTO-GONZALEZ, D. A.; PANHOCA, I. Correlation between the clinical phonoaudiological assessment and electromyographic activity of the masseter muscle. Journal of Applied Oral Science, v. 13, 4, p. 424-30, 2005.

BOLZAN, M. J. A. Análise da transformada em ondeletas aplicada em sinal geofísico. 
Revista Brasileira de Ensino de Física, v. 26, n. 1, p. 37-41, 2004.

CALIFANO, A. R. Análise Eletromiográfica do Músculo Masseter e Feixe Anterior do Músculo Temporal após Indução de Fadiga Muscular e Aplicação de LED (640 nm). 2006. 96 F. Dissertação (Mestrado em Engenharia Biomédica). Instituto de Pesquisa e Desenvolvimento. Universidade do Vale do Paraíba, São José dos Campos, 2006.

CALIFANO, A. R. et al. Electromyographic analysis of the masseter muscle and anterior bundle of the temporal muscle after muscular fatigue induction and LED irradiation (640 nm). Jornal Brasileiro de Laser, v. 2, p. 30-38, 2009.

DELAGI, E. F. et al. Anatomic Guide for the Electromyographer. Springfield, Illinois: Charles C. Thomas Publisher, 2005.

DELFINO, M. M. Análise da variabilidade da frequência cardíaca durante a caminhada em estreira rolante, em diferentes inclinações (declive e aclive) em indivíduos na faixa etária de 50 a 70 anos, utilizando a transformada wavelet contínua. 2006. São José dos Campos, 2006. Dissertação (Mestrado em Ciências Biológicas) - Instituto de Pesquisa e Desenvolvimento. Universidade do Vale do Paraíba, São José dos Campos, 2006.

DRAKE, R. L. et al. Gray's anatomia para estudantes. Rio de Janeiro: Elsevier, 2005. 1058p. ISBN 8535216383.

GONÇALVES, M.; SILVA, S. R. D. Análise de variáveis eletromiográficas durante contração isométrica fadigante. Salusvita, Bauru, v. 26, n. 1, p. 39-51, 2007.

GONZÁLEZ, I. M. et al. New Wavelet Indices to Assess Muscle Fatigue during Dynamic
Contractions. Engineering and Technology, v. 55, p. 480-485, 2009.

HANNINEN, O. et al. On-line determination of anaerobic threshold with rms-EMG. Biomedica biochimica acta, v. 48, n. 5-6, p. S493-S503, 1989.

KELENCZ, C. A. et al. Effect of Low-Power Gallium-Aluminum-Arsenium Noncoherent Light $(640 \mathrm{~nm})$ on Muscle Activity: A Clinical Study. Photomedicine and Laser Surgery, v. 28, n. 5, p. 647-652, Oct. 2010.

KUMAR, D. K.; PAH, N. D.; BRADLEY, A. Wavelet analysis of surface electromyography to determine muscle fatigue IEEE transactions on neural systems and rehabilitation engineering., v. 11, n. 4, p. 400-406, Dec. 2003.

MIYASHITA, M.; KANEHISA, H.; NEMOTO, I. EMG related to anaerobic threshold. Journal of Sports Medicine and Physical Fitness, v. 21, n. 3, p. 209-217, 1981.

MORITANI, T.; TAKAISHI, T.; MATSUMOTO, T. Determination of maximal power output at neuromuscular fatigue threshold. Journal of Applied Physiology, v. 74, n. 4, p. 1729-34, 1993.

MOTTA, A. J. C. M. Transformada Wavelet Aplicada a Sinais Eletromiográficos de Músculo Temporal em Contração Isométrica Máxima e Fadiga. 2009. 91f. Dissertação (Mestrado, Engenharia Biomédica) Instituto de Pesquisa e Desenvolvimento, Universidade do Vale do Paraíba, São José dos Campos, 2009.

MUÑOZ, I. S. S. et al. Análise Eletromiográfica do Músculo Masseter após Indução de Fadiga com Aplicação de Laser de Baixa Potência - Resultados Preliminares. Terapia Manual, v. 7, n. 31, p. 197-201, 2009. 
MUÑOZ, I. S. S. Transformada Wavelet Proceedings... Jyvaskyla, Finland: ISB, Aplicada a Sinais Eletromiográficos de 1995.

Músculo Masseter em Contração Isométrica Máxima e Fadiga. 2009. 115f. Dissertação (Mestrado em Engenharia Biomédica); Instituto de Pesquisa e Desenvolvimento. Universidade do Vale do Paraíba, São José dos Campos, 2009.

NOZAKI, S. et al. Range of motion exercise of temporo-mandibular joint with hot pack increases occlusal force in patients with Duchenne muscular dystrophy. Acta Myologica, v. 29, n. 3, p. 392-72, Dec. 2010.

PINTO NETO, O.; MAGINI, M. Electromiographic and kinematic characteristics of Kung $\mathrm{Fu}$ YauMan pam strike. Journal of Electromyogrphy and Kinesiology, v. 18, n. 6, p. 1047-1052, 2008.

SALOMONOW, M. A. Pratical Guide to Electromyography. In: INTERNATIONAL SOCIETY OF BIOMECHANICS CONGRESS, 15., $1995 . \quad$ JyVaskyla.

SHINOZAKI, E. B. Eficiência do laser terapia de baixa potência no tratamento das disfunções temporomandibulares. 2006. 112p. Dissertação (Mestrado em Bioengenharia) Universidade do Vale do Paraíba, São José dos Campos-SP; 2006.

TORRENCE, C.; COMPO, G. P. A Practical Guide to Wavelet analysis. Bulletin of the American Meteorological Society, v. 79, n. 1, p. 61-78, 1998.

TRIBESS, S.; VIRTUOSO JR., J. S. Prescrição de Exercícios para Idosos. Revista Saúde.com, v. 1, n. 2, p. 163-172, 2005.

VITTI, M.; BASMAJIAN, J. V. Integrated action of masticatory muscle: simultaneous EMG from eight intramuscular electrodes. The Anatomical Record, v. 187, n. 2, p. 173189, Feb. 1977. 